\title{
An efficient simulator of 454 data using configurable statistical models
}

Fredrik Lysholm ${ }^{1 *}$, Björn Andersson ${ }^{2}$ and Bengt Persson ${ }^{1,2}$

\begin{abstract}
Background: Roche 454 is one of the major $2^{\text {nd }}$ generation sequencing platforms. The particular characteristics of 454 sequence data pose new challenges for bioinformatic analyses, e.g. assembly and alignment search algorithms. Simulation of these data is therefore useful, in order to further assess how bioinformatic applications and algorithms handle 454 data.

Findings: We developed a new application named 454sim for simulation of 454 data at high speed and accuracy. The program is multi-thread capable and is available as $\mathrm{C}++$ source code or pre-compiled binaries. Sequence reads are simulated by $454 \mathrm{sim}$ using a set of statistical models for each chemistry. 454 sim simulates recorded peak intensities, peak quality deterioration and it calculates quality values. All three generations of the Roche 454 chemistry ('GS20', 'GS FLX' and 'Titanium') are supported and defined in external text files for easy access and tweaking.

Conclusions: We present a new platform independent application named 454sim. 454sim is generally 200 times faster compared to previous programs and it allows for simple adjustments of the statistical models. These improvements make it possible to carry out more complex and rigorous algorithm evaluations in a reasonable time scale.
\end{abstract}

\section{Introduction}

The introduction of $2^{\text {nd }}$ generation sequencing techniques has resulted in a myriad of new large DNA sequencing projects, many of which have posed new challenges for bioinformatics. Roche 454 is one of the major $2^{\text {nd }}$ generation sequencing platforms. The 454 instruments use a type of pyrosequencing chemistry, where the complementary strand is elongated through repeated cycles, each using one of the four nucleotides (flows). The complementary strand is elongated in the absence of a terminator, and homopolymer lengths are estimated by the light intensity recorded. As a consequence, homopolymer length uncertainties sometimes occur, especially at long homopolymer stretches [1]. This specific aspect of 454 data poses new challenges for downstream bioinformatic applications, such as assembly and alignment search algorithms [2-4]. Simulation of sequence data is an important and extensively used tool for assessing how bioinformatic applications and algorithms handle sequence data. The first software that was

\footnotetext{
* Correspondence: frely@ifm.liu.se

'IFM Bioinformatics and SeRC (Swedish e-Science Research Centre), Linköping University, S-581 83 Linköping, Sweden

Full list of author information is available at the end of the article
}

developed for simulation of 454 data was MetaSIM [5], which provided simulated data using the statistical distributions suggested for 454 data by Margulies et al. [1] However, MetaSIM did not produce the raw data that many algorithms utilize and did not model the 'Titanium' chemistry. As a response to these limitations, Flowsim [6] was created. Flowsim produced 454 raw data and allowed for 'Titanium' reads to be produced. Flowsim accepted parameters that modify a particular setting, but all 454 chemistry generations supported by Flowsim were written in source code, which made small modifications complicated. Flowsim and MetaSIM did not produce detailed information of the simulation run, which would for example allow evaluation of correct homopolymer identification in an efficient manner. In order to overcome these limitations, we now introduce 454sim.

\section{Methods and implementation}

$454 \mathrm{sim}$ generates simulated 454 data from input sequences in FASTA format. The algorithm models positive flows (a flow interpreted as one or more bases) with a normal distribution and negative flows (a flow interpreted as no base of that type, i.e. a noise flow) 
Table 1 454sim performance evaluation

\begin{tabular}{llll}
\hline Application & Reads/sec $^{(*)}$ & Wall time & CPU time \\
\hline 454sim 8-threads & 10,100 & $1 \mathrm{~min} 39 \mathrm{sec}$ & $11 \mathrm{~min} 30 \mathrm{sec}$ \\
454sim 1-thread & 2,730 & $6 \mathrm{~min} 6 \mathrm{sec}$ & $5 \mathrm{~min} 59 \mathrm{sec}$ \\
Flowsim 0.2.7 & 50.3 & $5 \mathrm{hrs} 31 \mathrm{~min} 40 \mathrm{sec}$ & $5 \mathrm{hrs} 30 \mathrm{~min} 20 \mathrm{sec}$ \\
\hline
\end{tabular}

Evaluation details for the '454sim' and 'flowsim-0.2.7' binary in simulation of 1,000,000 'Titanium' reads. The simulation was performed on a workstation equipped with an "Intel Core i7 920" processor, HT enabled, running Linux 64-bit. * Reads/sec is derived from wall time.

with a log-normal distribution [1]. This statistical model was used by both MetaSIM [5] and Flowsim [6], although $\mu$ and $\sigma$ for the distributions were chosen differently. In Flowsim, a degeneration model was also introduced, in which the standard deviation was gradually increased along the sequence. Flowsim also provided improved a calculation of quality values based on the statistical model by which flow-peak values were modelled. Both these improvements were also included in 454 sim and all parameters can easily be configured through a text format that describes the statistical model used for both flow-peak simulation as well as degradation of the standard deviation along the simulated read. The statistical models for 'GS20' and 'Titanium' that were present in Flowsim have been migrated to $454 \mathrm{sim}$, while $454 \mathrm{sim}$ also contains a model for 'GS FLX' reads.

454sim has been implemented using $\mathrm{C}++$ (compatible with GCC and the Intel compiler) to provide a highly efficient and multi-thread capable application. The program uses the Ziggurat algorithm [7] and the "Mersenne Twist Pseudorandom Number Generator Package" [8] to generate random variables with high speed and accuracy. The 454sim project is available as open source under the GNU General Public License. In order to facilitate modifications, it loads the statistical models for each 454 generation from separate text files. This text format defines a series of parameters in a 'key = value' syntax, which is easy to modify using any text editor. For more information regarding the generation parameter files as well as other available parameters, including their usage and examples, see http:// www.bioinfo.ifm.liu.se/454tools/454sim or https://sourceforge.net/p/bioinfo-454sim/.

\section{Evaluation}

In order to evaluate the performance of $454 \mathrm{sim}$, we simulated 1,000,000 'Titanium' reads using both $454 \mathrm{sim}$ and Flowsim, with default parameter settings, see Table 1. We did not test MetaSIM as it does not produce simulated raw data. For a 454 sim run utilizing only a single thread, a 50x increase in speed was achieved. If 454sim was executed using multiple threads, a 200x increase in speed was achieved. This translated to a reduction from approximately $51 / 2$ hours down to just over 6 minutes or 1 minute 39 seconds when using multiple threads, see Table 1.

\section{Discussion}

As the amount of sequence data produced keeps increasing, many downstream bioinformatic programs are already adapted to rapidly process large amounts of reads. In order to establish rigorous methods for the evaluation of for these programs, it is important and useful to also be able to simulate reads efficiently. Furthermore, as 454 data quality can vary, for example between metagenomic and genomic sequencing, it is also important to be able to modify the statistical models by which data is simulated. $454 \mathrm{sim}$ was constructed to meet these demands. The statistical models describing the Roche 454 platform chemistries are imported from separate text files. These can easily be modified by editing the file using a text editor. Entirely new models can also be added by creating an additional text file. To further facilitate analysis, $454 \mathrm{sim}$ also produces optional detailed output where the simulation of each base is described. This enables new types of evaluation such as correct homopolymer indel identification, which is not possible with previous tools. $454 \mathrm{sim}$ is at least two orders of magnitude faster than flowsim and reduced the run-time of a 'Titanium' chemistry simulation from 51 /2hours down to less than 2 minutes, on an Intel Core i7 920. 454sim is platform independent and available as $\mathrm{C}++$ source code [see Additional file 1] or the project homepage.

\section{Availability and requirements}

Project name: 454 sim

Project home page: https://sourceforge.net/p/bioinfo$454 \mathrm{sim} /$

Operating system(s): Platform independent

Programming language: $\mathrm{C}++$

Other requirements: -

License: GNU General Public License

Any restrictions to use by non-academics: -

\section{Additional material}

Additional file 1: Binaries and source code. The file contains Linux 32 and 64-bit binaries, a Windows 32-bit binary as well as source code. See the enclosed README for more information.

Acknowledgements and funding

We gratefully acknowledge financial support from the Swedish Research Council, the Research School of Medical Bioinformatics supported by the 


\section{Author details}

'IFM Bioinformatics and SeRC (Swedish e-Science Research Centre),

Linköping University, S-581 83 Linköping, Sweden. ${ }^{2}$ Department of Cell and Molecular Biology, Science for Life Laboratory, Karolinska Institutet, S-171 77 Stockholm, Sweden.

\section{Authors' contributions}

FL has implemented the software and written the manuscript. BP and BA have helped design the study and draft the manuscript. All authors read and approved the final manuscript.

\section{Competing interests}

The authors declare that they have no competing interests.

Received: 13 July 2011 Accepted: 26 October 2011

Published: 26 October 2011

\section{References}

1. Margulies M, Egholm M, Altman WE, Attiya S, Bader JS, Bemben LA, Berka J, Braverman MS, Chen YJ, Chen Z, Dewell SB, Du L, Fierro JM, Gomes XV, Godwin BC, He W, Helgesen S, Ho CH, Irzyk GP, Jando SC, Alenquer ML, Jarvie TP, Jirage KB, Kim JB, Knight JR, Lanza JR, Leamon JH, Lefkowitz SM, Lei M, Li J, et al: Genome sequencing in microfabricated high-density picolitre reactors. Nature 2005, 437:376-380.

2. Huse SM, Huber JA, Morrison HG, Sogin ML, Welch DM: Accuracy and quality of massively parallel DNA pyrosequencing. Genome Biol 2007, 8 : R143.

3. Gomez-Alvarez V, Teal TK, Schmidt TM: Systematic artifacts in metagenomes from complex microbial communities. ISME J 2009, 3:1314-1317.

4. Quince C, Lanzén A, Curtis TP, Davenport RJ, Hall N, Head IM, Read LF, Sloan WT: Accurate determination of microbial diversity from 454 pyrosequencing data. Nat Methods 2009, 6:639-641.

5. Richter DC, Ott F, Auch AF, Schmid R, Huson DH: MetaSim: a sequencing simulator for genomics and metagenomics. PLoS One 2008, 3:e3373.

6. Balzer S, Malde K, Lanzén A, Sharma A, Jonassen I: Characteristics of 454 pyrosequencing data - enabling realistic simulation with flowsim. Bioinformatics 2010, 26:i420-i425.

7. Marsaglia G, Tsang WW: The Ziggurat Method for Generating Random Variables. Journal of Statistical Software 2000, 5:1-7.

8. Matsumoto M, Nishimura T: Mersenne twister: a 623-dimensionally equidistributed uniform pseudo-random number generator. ACM Trans Model Comput Simulat 1998, 8:3-30.

doi:10.1186/1756-0500-4-449

Cite this article as: Lysholm et al: An efficient simulator of 454 data using configurable statistical models. BMC Research Notes 2011 4:449. 